\title{
Studying Star Formation with the Keck Interferometer
}

\author{
Rachel Akeson \\ Michelson Science Center, California Institute of Technology, Pasadena, \\ $C A, 91125, U S A$
}

\begin{abstract}
.
The Keck Interferometer utilizes the two 10-meter Keck telescopes as a direct detection interferometer in the infrared. The 85-meter baseline produces a fringe spacing of 5 milliarcseconds at 2 microns, or $0.7 \mathrm{AU}$ for sources in the Taurus star forming region. The first observations with the Keck Interferometer of $\mathrm{T}$ Tauri and Herbig Ae/Be sources are presented and the implications for circumstellar disk models are discussed.
\end{abstract}

\section{Introduction}

The sensitivity provided by using large aperture telescopes in optical/infrared interferometers allows observations of the milliarcsecond (mas) scale structure in young stellar objects (YSO's). Due to the high resolution required (1 AU subtends 7 mas at the distance to the Taurus Molecular Cloud), direct observations of the central region have been limited to date. Given the spatial resolution of current facilities, long-baseline infrared interferometry provides an ideal method for observing these inner regions, which are traced by material thermally emitting in the near-infrared.

Previous interferometric observations have resolved several types of young stellar objects: FU Ori, T Tauri and Herbig AeBe sources (see e.g., Malbet et al, 1998; Akeson et al, 2000; Millan-Gabet et al, 2001; Eisner et al, 2003). For the T Tauri and Herbig objects, the measured visibilities are generally not consistent with a geometrically flat disk with an inner radius less than a few stellar radii as predicted by spectral energy distribution fitting. Tuthill et al (2001) and Natta et al (2001) proposed that the inner radius is located at the destruction radius for directly heated dust, which can resolve this discrepancy. However, due to the sensitivity limits of the facilities used, the YSOs observed to date, particularly the $\mathrm{T}$ Tauris, are the brightest infrared examples and may not be representative. Here, I present the first observations of YSO's with the Keck Interferometer (KI). 


\section{Observing and data reduction}

The Keck Interferometer ${ }^{1}$ is a NASA-funded joint development among the Jet Propulsion Laboratory, the California Association for Research in Astronomy, and the California Institute of Technology, to interferometrically combine the two 10-m Keck telescopes for high sensitivity near-infrared visibility measurements $\left(\mathrm{V}^{2}\right)$, mid-infrared nulling interferometry at $10-12 \mu \mathrm{m}$, and differentialphase interferometry at $1.6-5 \mu \mathrm{m}$. A full description of the instrument is given by Colavita \& Wizinowich (2001).

The data presented here were taken in observations from June, 2002 to May, 2003. Data from the white-light channel $\left(\lambda_{\text {center }}=2.18 \mu \mathrm{m}\right.$ and $\left.\Delta \lambda \sim 0.3 \mu \mathrm{m}\right)$ are used. Observations consisted of a series of interleaved integrations on the source and several calibrators. The measured quantity is the visibility amplitude squared, which is an unbiased quantity, normalized such that an unresolved object has $\mathrm{V}^{2}=1.0$. The system visibility, the instrumental response to a point source, is measured with respect to calibrator stars, chosen to be close to the target source both in magnitude and on the sky. Further details on KI data reduction are available in Colavita et al (2003).

The field of view for the fringe tracker is 50 mas at $\mathrm{K}$. Within this field of view, several components may be contributing to the measured visibilities of YSO's: the stellar photosphere (unresolved in these observations), partially resolved emission from compact material (size less than the 5 mas fringe spacing) and extended emission which is completely resolved and contributes incoherently. In order to correctly interpret the partially resolved emission, presumably from the circumstellar disk, it is important to have accurate estimates for the stellar and extended components. The extended component may come from scattered light or other diffuse emission.

\section{Results}

\subsection{Observing program}

The Keck Interferometer is currently in a stage of engineering work and sharedrisk science observing. Four teams were competitively selected to work with the KI team for this period. Observations of YSO's were proposed by the team led by John Monnier and a few high mass objects were proposed by the team led by Bill Danchi. Along with scientists on the KI team, a program of observing and modeling $\mathrm{T}$ Tauri and Herbig sources has been pursued.

\subsection{Herbig Ae/Be stars}

A substantial number of Herbig Ae/Be sources have been observed at the InfraredOptical Telescope Array (Millan-Gabet et al, 2001) and at the Palomar Testbed Interferometer (PTI) (Eisner et al, 2003).

One of the goals of the KI Herbig program is to cover the complete range of spectral types for Herbig objects. Table 1 lists the Herbigs observed to date,

\footnotetext{
${ }^{1}$ Additional information at http://planetquest.jpl.nasa.gov/
} 
along with one FU Ori source. Reduction and modeling of this data is currently underway.

Table 1. Herbig sources observed to date

\begin{tabular}{lrr}
\hline Source & Spectral type & Notes \\
\hline V1685 Cyg & B2 & resolved \\
V1977 Cyg & B8 & resolved \\
HD 141569 & B9 & unresolved \\
AS 477 & A0 & resolved \\
MWC 758 & A7 & very resolved \\
HD 144432 & A9 & very resolved \\
V1057 Cyg & FU Ori & slightly resolved \\
\hline \hline
\end{tabular}

\subsection{T Tauri stars}

KI has observed two T Tauri stars to date: DG Tau and RW Aur. Both objects are resolved by the interferometer. No variation of the visibility with projected baseline or time is seen in the limited data collected so far. Neither source has a known binary companion, although speckle and adaptive optics searches are not sensitive to companions with milliarcsecond-scale separations. The observations of DG Tau are extensively discussed in Colavita et al (2003), including determination of the stellar and incoherent contributions to the measured visibility. Figure 1 shows the calibrated and system visibility points for DG Tau as observed on two separate nights at KI. For DG Tau, I use a fractional contribution of 0.41 from the stellar photosphere at $\mathrm{K}$ from the veiling observations of JohnsKrull et al (2001) and an incoherent contribution of 0.23 from the scattered light component measured in the lunar occultation observations of Leinert et al (1991). For RW Aur, the stellar fraction of 0.34 was computed using an effective temperature of $4590 \mathrm{~K}$ and a luminosity of $2.3 \mathrm{~L}_{\odot}$ (Hartigan et al, 1995).

For both sources, simple geometric models were used to estimate the size scale of the partially resolved component, believed to arise from the circumstellar disk. Table 2 lists the sizes for a uniform disk and ring model. The width of the ring is set by matching the excess $\mathrm{K}$ flux using a blackbody with a temperature of $1500 \mathrm{~K}$.

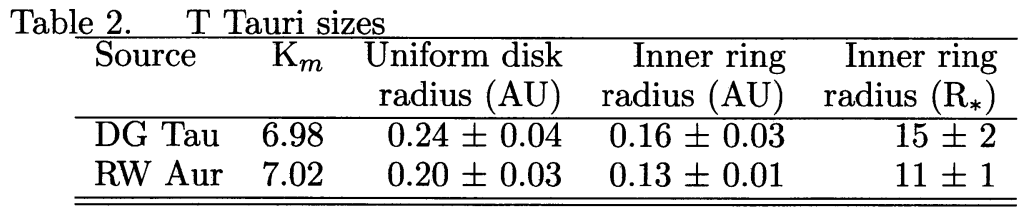




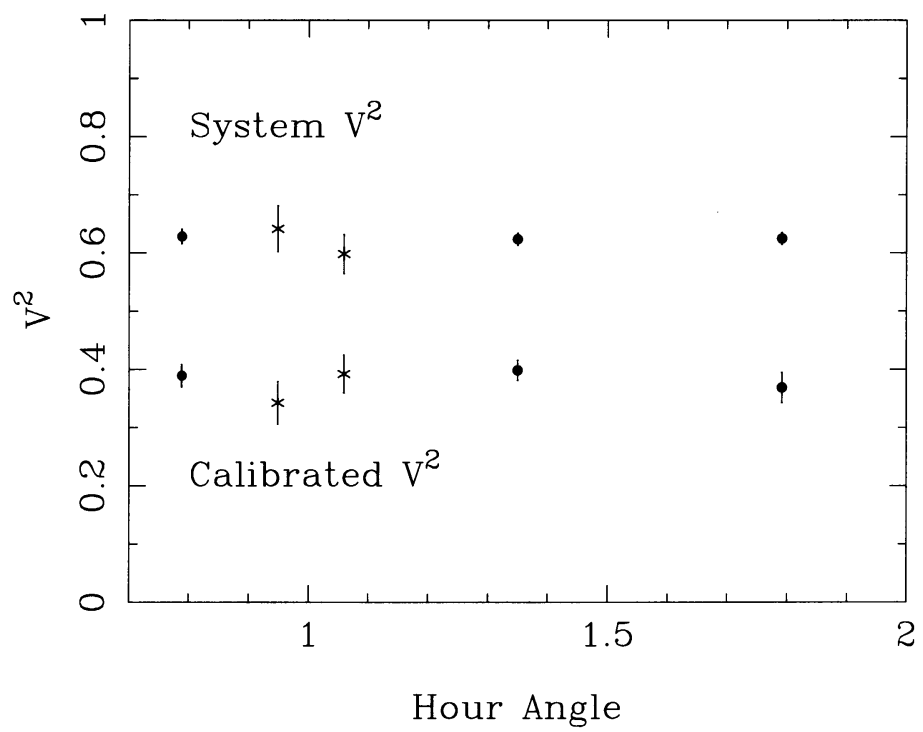

Figure 1. The calibrated visibility (bottom points) and measured system visibility (top points) for DG Tau. Data from the two nights of observations are plotted with different symbols.

\section{Discussion}

The size of the compact emission region of DG Tau and RW Aur is estimated to be a few tenths of AU given our estimates for the relative flux of the stellar and extended components. I assume that this component represents emission from a circumstellar disk and briefly discuss the implications for disk structure. The radii in Table 2 are similar to the sizes derived for the T Tauri stars $\mathrm{T}$ Tau $\mathrm{N}$ and SU Aur by Akeson et al (2000), and all are larger than predicted by models based on spectral energy distribution fitting. A ring model is used as a simple representation of models in which the inner disk radius corresponds to the dust destruction radius (Dullemond et al, 2001; Monnier \& Millan-Gabet, 2002). Figure 2 shows the radius corresponding to a dust destruction temperature $\left(\mathrm{T}_{\text {dust }}\right)$ of $1500 \mathrm{~K}$ plotted against the fit ring radius for DG Tau and RW Aur. In addition, four T Tauri sources observed at PTI are also plotted (Akeson et al, 2002).

In Figure 2, DG Tau is the only source for which the size implied by the measured visibility is significantly different from the predicted dust destruction radius. The caveats and possible sources of error in the measured size are discussed in detail by Colavita et al (2003). Recent modeling work by D'Alessio et al (this volume) includes both the stellar and the accretion luminosity in the calculation of the dust destruction radius, which pushes the dust destruction 
radius further from the star. For DG Tau, this results in a dust destruction radius of $\sim 0.2 \mathrm{AU}$, very similar to that measured with $\mathrm{KI}$.

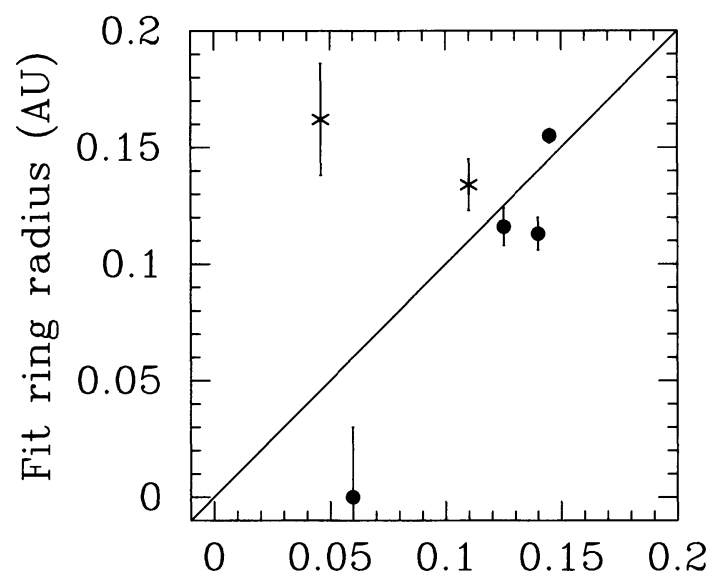

Dust destruction radius $(\mathrm{T}=1500 \mathrm{~K})(\mathrm{AU})$

Figure 2. The ring radius calculated from stellar properties for a dust destruction temperature of $1500 \mathrm{~K}$ compared to the fit ring radius for the two KI sources (stars) and four T Tauri stars observed at the Palomar Testbed Interferometer (circles). The straight line shows where these radii are equal.

Acknowledgments. The Keck Interferometer is funded by the National Aeronautics and Space Administration as part of its Navigator program. Part of this work was performed at the Jet Propulsion Laboratory, California Institute of Technology and the Michelson Science Center, under contract with NASA. Observations presented were obtained at the W.M. Keck Observatory, which is operated as a scientific partnership among the California Institute of Technology, the University of California and NASA. The Observatory was made possible by the generous financial support of the W.M. Keck Foundation. This work has made use of software produced by the Michelson Science Center at the California Institute of Technology. This work has made use of the SIMBAD database, operated at CDS, Strasbourg, France, and the NASA/IPAC Infrared Science Archive, operated by the JPL under contract with NASA.

\section{References}

Akeson, R. L., Ciardi, D. R., van Belle, G. T., Creech-Eakman, M. J., \& Lada, E. A. 2000, ApJ, 543, 313

Akeson, R. L., Ciardi, D. R., \& van Belle, G. T. 2002, Proceedings of the SPIE, 4838,1037 
Colavita, M. M. \& Wizinowich, P. L. 2002, Proceedings of the SPIE, 4838, 79 Colavita, M. et al. 2003, ApJ, 592, 83

Dullemond, C. P., Dominik, C., \& Natta, A. 2001, ApJ, 560, 957

Eisner, J. A., Lane, B. F., Akeson, R. L., Hillenbrand, L. A., \& Sargent, A. I. 2003, ApJ, 588, 360

Hartigan, P., Edwards, S., \& Ghandour, L. 1995, ApJ, 452, 736

Johns-Krull, C. M. \& Valenti, J. A. 2001, ApJ, 561, 1060

Leinert, C., Haas, M., Mundt, R., Richichi, A., \& Zinnecker, H. 1991, A\&A, 250, 407

Malbet, F. et al. 1998, ApJ, 507, L149

Millan-Gabet, R., Schloerb, F. P., \& Traub, W. A. 2001, ApJ, 546, 358

Monnier, J. D. \& Millan-Gabet, R. 2002, ApJ, 579, 694

Natta, A., Prusti, T., Neri, R., Wooden, D., Grinin, V. P., \& Mannings, V. 2001, A\&A, 371, 186

Tuthill, P. G., Monnier, J. D., \& Danchi, W. C. 2001, Nature, 409, 101 